\title{
Enhanced recovery care versus traditional care following osteotomies in developmental dysplasia of the hip in children: a retrospective case-cohort study
}

Jin Li

Wuhan Union Hospital

Saroj Rai

national academy of medical sciences

Renhao Ze

Wuhan Union Hospital

Xin Tang

Wuhan Union Hospital

Ruikang Liu

Huazhong University of Science and Technology

Pan Hong ( $\nabla$ hongpan2013@foxmail.com )

Wuhan Union Hospital https://orcid.org/0000-0003-2674-3016

\section{Research article}

Keywords: enhanced recovery after surgery, developmental dysplasia of the hip, complications, hospitalization, osteotomy

Posted Date: January 17th, 2020

DOI: https://doi.org/10.21203/rs.2.21179/v1

License: (c) (i) This work is licensed under a Creative Commons Attribution 4.0 International License. Read Full License

Version of Record: A version of this preprint was published at BMC Musculoskeletal Disorders on April 13th, 2020. See the published version at https://doi.org/10.1186/s12891-020-03243-z. 


\section{Abstract}

Background: Enhanced recovery after surgery (ERAS) has been shown to shorten the length of hospital stay and reduce the incidence of perioperative complications in many surgical fields. However, there has been a paucity of research examining the application of ERAS in major pediatric orthopedic surgeries. This study aims to compare the perioperative complications and length of hospital stay after osteotomies in children of developmental dysplasia of the hip (DDH) between ERAS and traditional care group.

Methods: The ERAS group consisted of 86 patients included in the ERAS program from January 2016 to December 2017. The Control group consisted of 82 DDH patients who received osteotomies from January 2014 to December 2015. Length of hospital stay, physiological function, postoperative visual analogue scale (VAS) score, and postoperative complications were compared between the two groups.

Results: The mean duration of hospital stay was significantly reduced from $10.0 \pm 3.1$ in the traditional care group to $6.0 \pm 0.8$ days in the ERAS $(P<0.001)$. Pain response was better in the ERAS group than traditional care group in terms of mean 3-day VAS score ( $2.9 \pm 0.8$ vs. $4.0 \pm 0.8, P<0.001)$ and maximum VAS score ( $4.1 \pm 0.8$ vs. $4.9 \pm 0.8, P<0.001)$. However, there was no significant difference in the frequency of break-out pain (VAS $>4$ ) between two groups $(29.5 \pm 6.3$ vs.30.6 $\pm 6.5, P=0.276)$. The frequency of fever is lower in the ERAS group, possibly due to better temperature monitoring and management in the operating room. The frequency of urinary infection in both groups were not noticeable because the catheter was removed promptly after the surgery.

Conclusion: The ERAS protocol is both safe and feasible for pediatric DDH patients undergoing osteotomies, and it can shorten the length of hospital stay without increasing the risk of perioperative complications.

\section{Background}

Minimal invasive surgery (MIS), damage control theory (DCT), and enhanced recovery after surgery (ERAS) are three breakthroughs in the 21st century[1]. ERAS was proposed by Henrik Kehlet in the 1990 s[2], and it has been widely applied in many surgical specialties for more than 20 years. The ERAS protocol is an evidence-based multidisciplinary perioperative approach, aiming to reduce the hospital stay, decrease the incidence of surgery-related complications, and promote early rehabilitation[3]. In orthopedic surgery, ERAS was adopted in high-volume standard procedures, such as primary hip and knee replacements. There are reports that the ERAS protocol is both safe and effective in orthopedic surgeries $[4,5,6]$. A meta-analysis showed that ERAS could facilitate the rapid recovery of physiological function, reduce the incidence of perioperative complications, shorten the duration of hospital stay[7].

However, there has been a paucity of literature examining the application of ERAS in major orthopedic surgeries in children. 
Developmental Dysplasia of the Hip (DDH) is a common bone and joint deformity of lower limbs in the pediatric population[8]. The incidence of DDH is 1.5 to 2.5 cases per 1000 live births.[9] The DDH treatment aims to achieve concentric hip reduction and painless hip joint in the adolescence and adulthood. Pavlik harness is an effective option for children younger than 6 months with DDH.[9] However, if diagnosed at walking age, the treatment strategy might be complex and controversial[10].

Pelvic osteotomy with or without femoral osteotomy is the mainstream surgical treatment for DDH[11]. The choice of osteotomy is mainly based on the age at the time of surgery, pathological and anatomical characteristics, severity of the dislocation, and clinical experience and preference of the surgeon. Osteotomy is the only effective method of treatment of DDH in patients older than 18 months [12]. However, it is not without complications. The most common perioperative complications include infection, hematoma, wound dehiscence leading to an extended hospital stay. Thus, establishing an ERAS protocol is urgent for major orthopedic surgeries in children. Since DDH is the most commonly performed elective surgery in our hospital, we firstly established and implemented an ERAS protocol for DDH in 2016.

This retrospective study aims to compare the incidences of complications and length of hospitalization between the ERAS group and the traditional care group. To our knowledge, this is the first ever study to evaluate the effectiveness of an ERAS protocol for DDH osteotomies in children.

\section{Methods}

\subsection{ERAS program}

Based on the ERAS protocol adopted in the pediatric surgery and orthopedics department, an ERAS protocol for pediatric orthopedic surgery was developed following a series of discussions with experts from pediatric orthopedic surgery, anesthesiology, pediatrics, and nurses from both the ward and operating room (OR). The protocol was then implemented for DDH patients undergoing surgical treatment from January 1, 2016.

The ERAS protocol for DDH osteotomies surgery included the following elements (Table 1, 2): preoperative education and counselling, antimicrobial prophylaxis, multi-mode analgesia, postoperative nausea and vomiting (PONV) prophylaxis, early oral food intake, less daily intravenous infusion volume, early removal of wound drainage and urinary catheters, early mobilization, and discharge criteria. Preoperative education and counselling included the aim and procedures of the ERAS protocol, pain coping strategies, parental expectation of this surgery, discharge criteria, and a follow-up plan. The fasting guideline requires cessation of clear fluid for at least 2 hours as well as solid foods for 6 hours before anesthesia[13]. The temperature of the operative room was controlled at 24 degrees, and warm fluids were used. Patients were administered with multimodal analgesia postoperatively including local infiltration of ropivacaine $(2.0 \mathrm{mg} / \mathrm{ml})$ around the incision, analgesia infusion pump based on the parental requirements, intravenous nonsteroidal anti-inflammatory (flurbiprofen, $2 \mathrm{~mm} / \mathrm{Kg}$ ) for 3 days, and then oral dexibuprofen oral suspension if necessary. Regular diet was advised after the patient was fully 
recovered from the anesthesia. A lower intravenous infusion volume was defined as less than $300 \mathrm{ml} / \mathrm{d}$ after surgery. The Foleys catheter was removed within 24 hours postoperatively. The drainage tube was recommended to be removed within 48 hours postoperatively. Early mobilization including on-bed movement of the healthy leg and upper extremities on postoperative day (POD) 1 . The antithrombotic prophylaxis was not necessary since children were at low risk of deep venous thrombosis (DVT). Discharge criteria were as follows: a visual analogue scale(VAS) score of $<3$ points with or without the use of oral analgesia; normal diet, no need for intravenous fluid; normal body temperature, no evidence of wound infection with normal complete blood count and C-reactive protein level; and no serious complications. We encouraged patient discharge once the discharge criteria were met.

\subsection{Population}

The hospital medical records of DDH patients receiving unilateral osteotomies in our institution were retrospectively reviewed. We included 82 patients in the traditional non-ERAS group or control group who underwent DDH surgery just prior to the implementation of the ERAS protocol (from January 2014 to December 2015). The ERAS group consisted of 86 patients who underwent DDH surgery after the implementation of the ERAS protocol (from January 2016 to December 2017). We enrolled the patients who underwent unilateral DDH osteotomies including one leg operation for bilateral DDH patients. Patients with prior neuromuscular disease, cerebral palsy, scoliosis and hyperlaxity were excluded from the study.

This study is approved by the Ethics Committee of Tongji Medical College, Huazhong University of Science and Technology.

\subsection{Surgical techniques}

All the patients underwent PO+PFO (See Fig. 1). Pelvic osteotomy is meant for increasing the acetabular coverage on femoral head. It includes reorientation osteotomies such as Salter, triple osteotomy, periacetabular osteotomy (PAO), and acetabuloplasty that changes the morphology such as Dega and Pemberton..Acetabuloplasty offers a higher rate of correction of acetabular dysplasia in comparison with reorientation osteotomies[14].

Pemberton osteotomy has been a preferred method of pelvic osteotomy in our institution. Femoral osteotomies are designed to reorient the femoral head by derotation and increasing the varus in order to stabilize and stimulate acetabular development $[15,16]$. Proximal femoral plate (PHP) system is used to fixate the osteotomy at the femoral side.

After the surgery, all patients were immobilized in Spica cast for 6 weeks until first out-patient visit.

\subsection{Clinical evaluation variables}

Physiological function outcome indicators, such as the first time that the patient was able to eat, defecate, as well as the removal time of the Foleys catheter and drainage tubes, and duration of hospital 
stay were recorded.

The postoperative pain response after surgery were recorded, including the average VAS score, the highest VAS score, and the frequency of break-out pain (VAS $\geq 4$ ). The incidence of all postoperative complications was recorded including nausea and vomiting, fever.

Statistical analysis

All descriptive data were presented as the mean \pm SD. Statistical analysis was performed using SPSS (SPSS Inc, Chicago, IL). P value of $<0.05$ is regarded as statistical significance.

\section{Results}

\subsection{Demographic data}

Demographic data are shown in Table 3. There were no significant differences in age, sex, surgical duration, intraoperative blood loss, and other comorbidities between the traditional non-ERAS group and ERAS group.

\subsection{Clinical outcomes}

The removal of Foleys and drainage tubes were significantly earlier in ERAS group than that in the traditional non-ERAS group (Table 4). The mean duration of hospital stay was significantly reduced from $10.0 \pm 3.1$ in the traditional non-ERAS group to $6.0 \pm 0.8$ days in the ERAS $(P<0.001)$.

Pain response was better in the ERAS group than traditional non-ERAS group in terms of mean 3-day VAS score $(2.9 \pm 0.8$ vs. $4.0 \pm 0.8, P<0.001)$ and maximum VAS score $(4.1 \pm 0.8$ vs. $4.9 \pm 0.8, P<0.001)$ (Table 5$)$. However, there was no significant difference in the frequency of break-out pain (VAS $>4$ ) between two groups (29.5 \pm 6.3 vs.30.6 $\pm 6.5, P=0.276)$.

Table 6 shows the surgery-related complications in both groups. There were no serious complications observed in this study. PONV was more common in the ERAS group as we used opiates as analgesics in the infusion pump. However, fever was less in the ERAS group, which possible due to better temperature monitoring and management in the operating room. No patients had postoperative urinary tract infection.

\subsection{Compliance with ERAS core components}

Compliance with the core components of ERAS protocol is shown in Table 7. During the perioperative period, the fasting time before surgery was shortened in ERAS group. The application of local analgesia, oral analgesia, PONV prophylaxis was well executed in ERAS group. Because of the possible adverse reactions of opiates (sufentanil or oxycodone), the application of analgesia infusion pump were not well executed. In all patients, oral dexibuprofen was given whenever necessary. Compliance of early removal of the Foleys catheter and drainage tubes in the ERAS group were $100 \%$ and $95.3 \%$ respectively. The most common cause of delayed removal of drainage catheters was high drainage volume on POD2, which 
may increase the risk of postoperative hematoma and local infection. Compliance with early regular diet was $95.3 \%$.

\section{Discussion}

ERAS protocol has been widely applied in many surgical fields, especially in abdominal surgery. In the past few years, there were reports on its application in orthopedic surgery such as joint replacement, trauma and spine surgery $[17,18,19]$. However, the literature on pediatric orthopedic surgery was quite rare. Pediatric population is a group that requires special attention and discretion.

Preoperative evaluation of malnutrition and anemia are critical as they are associated with a higher rate of postoperative complications such as delayed wound healing, infection, and prolonged duration of hospital stay[20,21]. A recent history of common cold, cough, diarrhea should be taken carefully before admission; if one had such symptoms, surgery should be postponed. A preoperative hemoglobin level of less than $12 \mathrm{~g} / \mathrm{dL}$ should be investigated to rule out other pathological causes. If the body weight is 2 standard deviation lighter than the standard weight in peers, more attention should be paid to the patients.

Preoperative counseling and education are crucial components of the ERAS protocol. Patients and patient's legal guardians should be counseled and educated for the expectation adjustment, pain coping mechanism, and the rehabilitation plan preoperatively. A team consisting of surgeons, nurses, physical therapists should discuss the treatment strategy with the legal guardians thoroughly. Although preoperative education reduces the level of anxiety in parents, it does not reduce the duration of hospital stay significantly, lower the VAS score and the incidence of complications[22].

Multi-mode analgesia is advocated to boost the effect of pain alleviation and reduce the incidence and severity of adverse effects. One of the key aspects of ERAS program is to reduce the dose of opiates and even eliminate the application of opiates postoperatively [23]. Nonsteroidal anti-inflammatory drugs (NSAIDs) are the cornerstone for postoperative pain alleviation, but the possible sides effects to the digestive and cardiovascular system should not be overlooked. Dexibuprofen, a non-selective NSAID, has been widely used in pediatric orthopedics to cope with mild to moderate pain, but the long-term effects remain to be investigated. Unlike adults, most children are not able to endure pain. Novel drug such as bupivacaine liposome with longer half-life has been used in developed and used in clinical practice[24]. However, its application in children has not been thoroughly investigated. Dexamethasone is a strong anti-inflammatory drug, high dosage $(>0.1 \mathrm{mg} / \mathrm{kg})$ is able to alleviate all kinds of postoperative pain[25] De Oliveira GS et al. performed a meta-analysis of randomized controlled trials to evaluate the dosedependent analgesic effects of perioperative dexamethasone. They reported that an intraoperative infusion of $10 \mathrm{mg}$ mg dexamethasone could reduce the duration of hospital stay significantly, and it is not associated with delayed healing and infection [26]. However, the safety and validity of application of glucocorticoids in children remains to be investigated. 
Peripheral nerve block is routinely performed in patients with long bone fracture surgeries; however, it is not commonly performed in DDH patients. Local anesthetic agents such as lidocaine and ropivacaine have also been reported to use around the incision site for postoperative pain management. As for joint replacement surgeries, surgeons advocate the injection of a cocktail consisting of opiates, NSAIDs, and steroids periarticularly [27]. However, this technique has not been used and validated in our institute for the pediatric population.

American Society of Anesthesiology has recommended that the preoperative fasting for children should be at least 2 hours for clear water, 4 hours for breast milk, 6 hours for non-human or cow milk, 8 hours for meat or oily food. It has been validated that 2 hours fasting for clear water intake is safe in children, and it significantly reduces the thirst and hunger of sick children while waiting for the surgery [28]. In our institute, we try to calculate the precise timing of surgery and minimize the fasting time for children.

Most of the orthopaedic procedure does not need the drainage tub[29]; however, it is recommended in the DDH patients as the wounds are covered in Spica cast, and there is always the possibility of blood loss from the osteotomy sites causing postoperative hematoma and infection. In routine ERAS program, all kinds of catheters and drainage tubes are encouraged to remove early to promote early rehabilitation.

Early diet is recommended for DDH patients after anesthesia awareness. However, most young children are not able to eat regular diet immediately after surgery. Noodles or thin soup was recommended for young children on the first meal postoperatively. Food rich in dietary fiber is recommended for patient to lower the incidence of constipation. Walking is not possible postoperatively as the hips are immobilized in Spica cast. But early ambulation is always encouraged.

So far, there are limited reports of ERAS program in the field of pediatric orthopedics, most of our core components in the ERAS protocol were derivatives from research in adults. In clinical practice, the application of multi-mode analgesia alleviates the postoperative pain response that increases the surgical cost. The predictive increase of medication cost might be compensated by the reduction of hospital stay, however, that requires multi-center study.

\section{Conclusion}

The ERAS protocol is both safe and feasible choice for pediatric DDH patients undergoing osteotomies, and it shortens the duration of hospital stay. Continuous education and training should be given to the ERAS team, including the surgeon, nurses, anesthesiologist, caregiver, paediatrician to implement the protocols more strictly. Long-term follow up is required to validate the benefits of ERAS application in pediatric orthopaedics.

\section{Abbreviations}

ERAS = enhanced recovery after surgery, MIS = minimal invasive surgery, DCT = damage control theory, $\mathrm{POD}=$ postoperative day, $\mathrm{PONV}=$ postoperative nausea and vomiting, $\mathrm{VAS}=$ visual analog scale, $\mathrm{DDH}=$ 
developmental dysplasia of the hip, $\mathrm{PO}=$ pelvic osteotomy, $\mathrm{PFO}=$ proximal femoral osteotomy

\section{Declarations}

\section{Acknowledgements}

Not applicable.

\section{Funding}

None.

\section{Author's contributions}

$\mathrm{PH}^{*}$ is in charge of the main idea and is the guarantor of integrity of the entire clinical study; $\mathrm{JL}$ and $\mathrm{RHZ}$ are in charge of the study concepts, design, manuscript preparation and editing; PH and SR are in charge of the language polishing and the grammar revision; RKL and XT is in charge of the collection of the study data. All authors read and approved the final manuscript.

\section{Availability of data and material}

This is a report of comparative study. To protect privacy and respect confidentiality, no raw data have been made available in any public repository. The operation reports, imaging studies are all retained as per normal procedure within the medical records of our institution.

\section{Competing interests}

The authors declare that they have no competing interests.

\section{Ethics Approval and Consent to Participate}

Not applicable.

\section{Consent for publication}

Written consents were obtained from the patients for publication of this paper.

\section{References}

1. Kehlet H, Dahl JB. Anaesthesia, surgery and challenges in postoperative recovery [J]. Lancet, 2003, 362 (9399):1921-1928.

2. Kehlet $\mathrm{H}$. Multimodal approach to control postoperative pathophysiology and rehabilitation $[\mathrm{J}] . \mathrm{Br} \mathrm{J}$ Anaesth, 1997, 78(5): 606-617. 
3. Wilmore DW, Kehlet H.Management of patients in fast tract surgery [J]. BMJ, 2001, 322 (7284):473476.

4. Kehlet H. Fast-track hip and knee arthroplasty [J]. Lancet, 2013, 381(9878):1600-1602.

5. Lamplot JD, Wagner ER, Manning DW. Multimodal pain management in total knee arthroplasty: a prospective randomized controlled trial [J]. J Arthroplasty, 2013, 29(2): 329-334.

6. Khan SK, Malviya A, Muller SD, et al. Reduced short-term complications and mortality following enhanced recovery primary hip and knee arthroplasty: results from 6,000 consecutive procedures [J]. Acta Orthop, 2014, 85(1):26-31.

7. Hu ZC, He LJ, Chen D, et al. An enhanced recovery after surgery program in orthopedic surgery: a systematic review and meta-analysis [J]. J Orthop Surg Res, 2019, 14(1):77.

8. Davies SJ, Walker G. Problems in the early recognition of hip dysplasia [J]. J Bone Joint Surg Br. 1984; 66(4):479-484.

9. Gulati V, Eseonu K, Sayani J, et al. Developmental dysplasia of the hip in the newborn: A systematic review [J]. World J Orthop 2013; 4(2):32-41.

10. Bolland BJ, Wahed A, Al-Hallao S, et al. Late reduction in congenital dislocation of the hip and the need for secondary surgery: radiologic predictors and confounding variables [J]. J Pediatr Orthop 2010; 30:676-682.

11. Wang CW, Wang TM, Wu KW, et al. The comparative, long-term effect of the Salter osteotomy and Pemberton acetabuloplasty on pelvic height, scoliosis and functional outcome [J]. Bone Joint $J$ 2016; 98-B(8):1145-1150.

12. Luo $Y$, Wang $L$, Chen $M$, et al. Salter and Pemberton osteotomy for the treatment of developmental hip dysplasia [J]. Modern Instruments and Medical 2017; 23(4):21-23.

13. Practice guidelines for preoperative fasting and the use of pharmacologic agents to reduce the risk of pulmonary aspiration: application to healthy patients undergoing elective procedures: an updated report by the American Society of Anesthesiologists Committee on Standards and Practice Parameters. Anesthesiology 2011;114:495-511.

14. López-Carreño E, Carillo H, Gutiérrez M. Dega versus Salter osteotomy for the treatment of developmental dysplasia of the hip. J Pediatr Orthop B 2008;17(5):213-221.

15. Moraleda L, Bravo C, Forriol F, Albiñana J. Does orientation of the femoral head affect acetabular development? An experimental study in lamb. J Pediatr Orthop 2017;1.

16. Blockey NJ. Derotation osteotomy in the management of congenital dislocation of the hip. J Bone Joint Surg [Br] 1984;66(4):485-490.

17. Wang MY, Chang PY, Grossman J. Development of an enhanced recovery after surgery (ERAS) approach for lumbar spinal fusion[J]. J Neurosurg Spine, 2017, 26(4):411-418.

18. Mcdonald DA, Siegmeth R, Deakin AH, et al. An enhanced recovery programme for primary total knee arthroplasty in the United Kingdom-follow up at one year[J]. Knee, 2012, 19(5): 525-529. 
19. Talboys R, Mak M, Modi N, et al. Enhanced recovery programme reduces opiate consumption in hip hemiarthroplasty [J]. Eur J Orthop Surg Traumatol, 2016, 26(2):177-181.

20. Beattie WS, Karkouti K, Wijeysundera DN, et al. Risk associated with preoperative anemia in noncardiac surgery: a single-center cohort study[J]. Anesthesiology, 2009, 110(3):574-581.

21. Berend KR, Lombardi AV, Mallory TH. Rapid recovery protocol for peri-operative care of total hip and total knee arthroplasty patients [J]. Surg Technol Int, 2004, 13(2):239-247.

22. Kearney M, Jennrich MK, Lyons S, et al. Effects of preoperative education on patient outcomes after joint replacement surgery[J]. Ortho Nurs, 2011, 30(6):391-396.

23. Chalmers DJ, Bielsky A, Wild TT, et al. Continuous local anesthetic infusion for children with spina bifida undergoing major reconstruction of the lower urinary tract[J].J Pediatr Urol, 2015, 11(2):e1-e5.

24. Mont MA, Beaver WB, Dysart SH, et al. Local infiltration analgesia with liposomal bupivacaine improves pain scores and reduces opioid use after total knee arthroplasty: results of a randomized controlled trials [J]. J Arthroplasty, 2018, 33(1): 90-96.

25. Backes JR, Bentley JC, Politi JR, et al. Dexamethasone reduces length of hospitalization and improves postoperative pain and nausea after total joint arthroplasty: a prospective, randomized controlled trails [J]. J Arthroplasty, 2013, 28(8 suppl):11-17.

26. De Oliveira GS, Almeida MD, Benzon HT, et al. Perioperative single dose systemic dexamethasone for postoperative pain: a meta-analysis of randomized controlled trials [J].Anesthesiology, 2011, 115(3):575-588.

27. Brady M, Kinn S, Ness V, et al. Preoperative fasting for preventing perioperative complications in children [J]. Cochrane Database Syst Rev, 2009, 7(4): CD005285.

28. Reynolds RA, Jackson H. Concept of treatment in supracondylar humeral fractures [J]. Injury, 2004.12.013

29. Frye BD, Hannon P, Santoni BG, et al. Drains are not beneficial in primary shoulder arthroplasty[J]. Orthopedics. 2019,42(1):e29-e31.

\section{Tables}

Table 1. The ERAS protocol versus traditional care

\begin{tabular}{|l|l|l|}
\hline & $\begin{array}{l}\text { Traditional care } \\
\text { protocol }\end{array}$ & ERAS protocol \\
\hline Psychological counseling & Informed consent & Education and illustration \\
\hline Nutrition and Anemia Evaluation & No requirement & Proper assessment \\
\hline $\begin{array}{l}\text { Preoperative fasting } \\
\text { PONV prophylaxis }\end{array}$ & Fasting 12h, water 4h & Fasting 6h, water 2h \\
\hline $\begin{array}{l}\text { Postoperative diet Analgesic } \\
\text { Perioperataive }\end{array}$ & No requirement & 5-HT receptor antagonist \\
$\begin{array}{l}\text { management } \\
\text { Removal of urinary catheter }\end{array}$ & No requirement & $\begin{array}{l}\text { Regular diet after anesthesia } \\
\text { awareness }\end{array}$ \\
\hline \begin{tabular}{l} 
Removal of wound drainage \\
\hline
\end{tabular} & POD 2-3 & Comprehensive analgesic regimen \\
\hline
\end{tabular}

Table 2. Components of ERAS pathway for DDH 


\begin{tabular}{l|l|l|}
\hline Preoperative & Intraoperative & Postoperative \\
\hline Parental education & Local anesthesia of incision & Analgesia infusion pump \\
\hline Evaluation of nutrition & Maintenance of body temperature & Early diet after anesthesia awareness \\
\hline No bowel preparation & Maintenance of blood volume & PONV prophylaxis \\
\hline No prolonged fasting & & Early removal of catheters \\
\hline Preventive antibiotics & & Compliance and follow-up \\
\hline Carbohydrate loading & & \\
\hline
\end{tabular}

Table 3. Demographic characteristics

\begin{tabular}{l|l|l|l|}
\hline Category & Traditional Care $(\mathrm{n}=82)$ & ERAS $(\mathrm{n}=86)$ & P Value \\
\hline Average age & $4.2 \pm 1.3$ & $4.0 \pm 1.3$ & 0.515 \\
\hline Gender & 13 & 11 & \\
\hline Male & 69 & 75 & 0.358 \\
\hline Female & $23.28 \pm 1.45$ & $23.62 \pm 1.45$ & 0.138 \\
\hline BMI (mean) & $106.4 \pm 8.7$ & $104.2 \pm 8.9$ & 0.148 \\
\hline Operative time (min) & $144.1 \pm 28.1$ & $146.9 \pm 28.6$ & 0.538 \\
\hline
\end{tabular}

$\mathrm{BMI}=$ body mass index

Table 4 Postoperative physiological function outcomes

\begin{tabular}{|l|l|l|l|}
\hline & Traditional Care & ERAS & p value \\
\hline First eating time, $\mathrm{h}$ & $12.2 \pm 1.5$ & $5.2 \pm 0.8$ & 0.000 \\
\hline Urinary catheter removal, $\mathrm{h}$ & $54.2 \pm 3.8$ & $10.0 \pm 1.5$ & 0.000 \\
\hline Wound drainage removal, $\mathrm{h}$ & $41.9 \pm 3.7$ & $29.3 \pm 3.6$ & 0.000 \\
\hline First defecation,h & $4.4 \pm 1.1$ & $4.4 \pm 1.1$ & 0.869 \\
\hline Length of hospital stay, d & $10.0 \pm 3.1$ & $6.0 \pm 0.8$ & 0.000 \\
\hline
\end{tabular}

Table 5 VAS pain score of 3-d after surgery

\begin{tabular}{l|l|l|l|}
\hline & Traditional Care & ERAS & p value \\
\hline Mean VAS score & $4.0 \pm 0.8$ & $2.9 \pm 0.8$ & 0.000 \\
\hline Maximum VAS score & $4.9 \pm 0.8$ & $4.1 \pm 0.8$ & 0.000 \\
\hline Outbreak pain (VAS $\geq 4)$ & $30.6 \pm 6.5$ & $29.5 \pm 6.3$ & 0.276 \\
\hline
\end{tabular}

Table 6 Postoperative complications and adverse reactions

\begin{tabular}{l|l|l|l|}
\hline & Traditional Care $(\mathrm{n}=82)$ & ERAS $(\mathrm{n}=86)$ & $\mathrm{p}$ value \\
\hline Nausea and vomiting & 38 & 63 & 0.000 \\
\hline Fever & $1.5 \pm 1.1$ & $1.0 \pm 0.9$ & 0.002 \\
\hline Urinary infection & 0 & 0 & 0.663 \\
\hline
\end{tabular}

Table 7 Compliance with ERAS core components in ERAS group

\begin{tabular}{l|l|l|}
\hline & No. following protocol & N\% \\
\hline Preoperative education and counseling & 86 & $100 \%$ \\
\hline Preoperative fasting 6 h, clear water 2 $\mathrm{h}$ & 86 & $100 \%$ \\
\hline Multimodal analgesia & 86 & $100 \%$ \\
\hline Local anesthesia of incision & 65 & $75.6 \%$ \\
\hline Analgesia infusion pump & 86 & $100 \%$ \\
\hline Scheduled intravenous analgesia & 86 & $100 \%$ \\
\hline Oral analgesia & 86 & $100 \%$ \\
\hline PONV prophylaxis & 82 & $95.3 \%$ \\
\hline Early diet & 86 & $100 \%$ \\
\hline Removal of Urinary catheter on POD 1 & 82 & $95.3 \%$ \\
\hline Removal of wound drainage on POD2 & \multicolumn{2}{|l}{}
\end{tabular}


Figures

\section{Figure 1}

PO+PFO in of left Hip of 5-year-old girl: a. AP view of the pelvis before the surgery b. AP view of the pelvis after the surgery $\mathrm{c}$. full-length images of the lower extremities at 3 months follow-up visit 